Psychoneuroendocrinology. 2015 May ; 55: 164-172. doi:10.1016/j.psyneuen.2015.02.009.

\title{
Oxytocin and HPA stress axis reactivity in postpartum women
}

\author{
E.Q. Cox ${ }^{a},{ }^{,}$, A. Stuebe ${ }^{b}$, B. Pearson ${ }^{a}$, K. Grewen ${ }^{a}$, D. Rubinow ${ }^{a}$, and S. Meltzer-Brody ${ }^{a}$ \\ aDepartment of Psychiatry, University of North Carolina at Chapel Hill, Chapel Hill, NC 27599- \\ 7160, United States \\ ${ }^{b}$ Department of Obstetrics and Gynecology, University of North Carolina at Chapel Hill, Chapel \\ Hill, NC 27599-7160, United States
}

\section{Summary}

Background-Lactation is thought to buffer stress reactivity via oxytocin (OT). Dysregulation of the HPA axis has been reported in women with postpartum depression (PPD). The cooccurrence of PPD and lactation failure suggests that abnormalities in OT signaling may play a role in PPD. We hypothesized that abnormal OT signaling is implicated in dysregulated HPA axis reactivity among postpartum women with mood symptoms. In a prospective perinatal cohort, we tested associations between OT levels during breastfeeding and stress reactivity.

Methods-We recruited 52 pregnant women who intended to breastfeed, among whom 47 underwent a standardized stressor, the Trier Social Stress Test (TSST), at 8 weeks postpartum. 39 were breastfeeding at time of TSST. We assessed mood symptoms using validated instruments and defined as symptomatic women with EPDS $\geq 10$ and/or Spielberger $\geq 34$. Following IV placement for blood draws, women breastfed their infants and then underwent the TSST. Mothers' hormone responses were quantified.

Results-Among symptomatic breastfeeding women ( $N=11$; asymptomatic $N=28)$, we found lower OT levels during breastfeeding $(p<0.05)$ and higher CORT levels $(p<0.05)$ both during breastfeeding and the TSST, as compared to asymptomatic breastfeeding women. In a mixed effects model examining CORT reactivity by symptom group and OT AUC, we observed a paradoxical response in symptomatic breastfeeding women during the TSST (group $\times$ time $\times$ OT AUC $p<0.05$ ); higher OT AUC was associated with higher CORT.

Conclusions-In all breastfeeding women, the surge of OT during feeding appears to buffer subsequent stress-induced CORT secretion. However, in symptomatic breastfeeding women, we found a positive correlation between OT AUC and CORT, instead of the expected negative correlation, which we found among asymptomatic women.

\section{Keywords}

Pospartum depression; Lactation failure; HPA axis; Oxytocin; Cortisol

(C) 2015 Published by Elsevier Ltd.

*Corresponding author. Tel.: +1 919966 8702; fax: +1 919966 9646., elizabeth.q.cox@gmail.com, ecox@unch.unc.edu (E.Q. Cox). Conflict of interest statement

None declared. 


\section{Introduction}

Postpartum depression (PPD) and early, undesired weaning attributed to lactation dysfunction (disrupted lactation) are two common public health problems following childbirth. PPD is defined by the DSM-5 as a major depressive episode with onset beginning during pregnancy or within the first 4 weeks postpartum (American Psychiatric Association, 2013). PPD affects between 10 and $15 \%$ of all mothers and is the greatest risk factor for maternal suicide and infanticide (Lindahl et al., 2005). PPD is often accompanied by severe anxiety or ruminating thoughts (Bernstein et al., 2008; Putnam et al., in press). It has also been shown to significantly compromise mother-infant attachment and impairs maternal implementation of recommended parenting practices that provide a safe and stimulating environment for appropriate child growth and development (Flynn et al., 2004).

Breastfeeding is recommended by all major medical groups for the first year of the child's life to protect the infant from infection and development of chronic diseases; however, early, undesired weaning attributed to problems with lactation physiology remains very common in the US and has been estimated to occur in one out of eight women (Stuebe et al., 2014). In addition to increased child morbidity, early weaning also impacts maternal health, as curtailed breastfeeding is associated with higher maternal rates of ovarian and breast cancers, type 2 diabetes, hypertension and myocardial infarction (AHRQ, 2007). PPD has been associated with undesired weaning including reduced breastfeeding duration and alterations in lactation physiology (Groer and Davis, 2006; Dennis and McQueen, 2009; Figueiredo et al., 2013; Kendall-Tackett et al., 2013; Paul et al., 2013). In a recent report that examined data from the Infant Feeding Practices II Study, early, undesired weaning was more common among women with symptoms of PPD, affecting $56 \%$ of women with depressive symptoms at 2 months postpartum, compared with $44 \%$ of women without depressive symptoms (Stuebe et al., 2014).

Oxytocin (OT) is a neuroendocrine hormone that is essential for normal breastfeeding physiology, as it stimulates breast myoepithelial cell contraction, which transfers milk to the areola for the infant (Pang and Hartmann, 2007). OT has been implicated in maternal behavior and in forming and maintaining social bonds, particularly in its interaction with dopamine (Pedersen et al., 1994; Pedersen, 1997; Numan et al., 2005; Aragona et al., 2006). In an investigation of lactating rats and maternal behavior, Shahrokh et al. demonstrated a direct effect of OT on dopamine release within the mesocorticolimibic dopamine system; OT neurons in the medial preoptic area (mPOA) were shown to regulate dopamine function in the ventral tegmental area (VTA), thereby determining the rewarding properties of a pup (Shahrokh et al., 2010). It is possible that with improper OT signaling, reward properties are altered, which could lead to changes in maternal behavior and mood.

The literature is small and conflicted regarding OT abnormalities in major depression, with studies showing that OT has different effects on different individuals (Meinlschmidt and Heim, 2007; Cyranowski et al., 2008; Parker et al., 2010; Chen et al., 2011; Mah et al., 2013; Thombs et al., 2014). Genetic variations in the OT receptor have been implicated in PPD and decreased breastfeeding duration (Jonas et al., 2013). Disruption of normal OT physiology has been linked to a dysregulated stress response and poor feeding outcomes in 
animal models (Lonstein and Stern, 1998). Recent work by our research group suggests that there is a relationship between symptoms of depression and anxiety and differences in OT response and effect during breastfeeding, thereby also implicating possible abnormalities in OT signaling in PPD (Stuebe et al., 2013).

In addition to the possible abnormalities in OT signaling, dysregulation of the hypothalamic pituitary adrenal (HPA) stress axis, including insufficient glucocorticoid signaling and impaired feedback regulation of relevant stress response systems, has been demonstrated in women with PPD (Greenwood and Parker, 1984; Magiakou et al., 1996; Wisner and Stowe, 1997; Bloch et al., 2003; Bloch et al., 2005; Groer and Davis, 2006; Jolley et al., 2007). For example, Jolley et al. studied postpartum women who underwent a physical stressor (maximal treadmill test) and measured mood, ACTH and cortisol (CORT) responses to the stressor. They reported a significant difference in within-subject ACTH levels predicting CORT regression slopes in postpartum women with and without depression at both 6 and 12 weeks postpartum; non-depressed postpartum patients exhibited a normal regulated feedback relationship of the HPA axis, with CORT levels rising in response to increasing levels of $\mathrm{ACTH}$, while depressed postpartum patients exhibit an atypical and dysregulated pattern whereby higher levels of ACTH did not result in increased levels of CORT (Jolley et al., 2007).

Prior work suggests that breastfeeding immediately before a social stressor buffers CORT response (Heinrichs et al., 2001). In male subjects, both OT and social support blunt HPA axis activation (Heinrichs et al., 2003). To date, no studies to our knowledge have quantified the relationship between the HPA axis, OT, and PPD symptoms. Thus, in the present study, we sought to assess the relationship between OT and HPA reactivity in breastfeeding women with and without postpartum depression symptoms. A well validated measurement of HPA stress reactivity is the Trier Social Stress Test (TSST), introduced by Kirschbaum et al. (1993) as a standardized protocol for inducing and studying the psychosocial stress response and dysregulation of the HPA axis in the laboratory setting (Kirschbaum et al., 1993). The TSST has been shown to reliably induce a well-characterized HPA axis response (Kudielka et al., 2007a,b). Therefore, we administered the TSST to symptomatic and asymptomatic lactating mothers. We hypothesized that the TSST would induce a differential stress response in mothers with symptoms of depression and anxiety versus euthymic mothers. We further hypothesized that the dysregulation of HPA stress reactivity in women with PPD may reflect deficient OT signaling, which could be assessed under breast feedinginduced stimulation and which might be reflected by the loss of expected associations between OT and CORT in women with PPD symptoms.

\section{Materials and methods}

We recruited women in the third trimester of pregnancy who intended to breastfeed for a longitudinal study of maternal mood and neuroendocrine response to infant feeding and psychosocial stress. The study has been described in detail elsewhere (Stuebe et al., 2013). We planned to recruit 25 women with current or past depression or anxiety disorders and 25 women with no such history. The study was approved by the UNC Institutional Review Board and all participants gave informed consent. Participants were recruited from prenatal 
obstetric and perinatal psychiatry clinics at University of North Carolina (UNC) Hospitals from February 2010 through February 2011. We included women who either had a current or past diagnosis of unipolar depression or an anxiety disorder (i.e., Generalized Anxiety Disorder, Obsessive Compulsive Disorder, Post-traumatic Stress Disorder) or no current or prior history of mood disorders, assessed by diagnostic interview. We excluded from participation women with (1) current tobacco or illicit substance use; (2) prenatal diagnoses that would interfere with breastfeeding; (3) endocrine disorders that might affect lactation or (4) chronic medication use that is contraindicated during lactation. Participants were assessed and diagnosed by a board-certified psychiatrist with extensive training in administration of structured research interviews (SMB) using the depression and anxiety disorder modules of the Structured Clinical Interview (SCID) (First et al., 2002).

At enrollment in the third trimester, participants provided a morning, non-fasting blood sample and completed the Edinburgh Postnatal Depression Scale (EPDS) (Cox et al., 1987) and the Spielberger State and Trait Anxiety Inventory (STAI) (Spielberger, 1983). The same instruments were completed at 2- and 8-weeks postpartum. A modified version of The Trier Social Stress Test (TSST) was administered at 8-weeks postpartum after participants fed or held their infants. Our objective was to quantify the acute effect of lactation on stress response; therefore, feeding status was classified based on how mothers chose to feed during the study visit. Participants were classified as "breastfeeding" if they either breastfeed their infant $(N=38)$ or expressed milk $(N=1)$ during the study visit; they were classified as "not breastfeeding" if they held $(N=2)$ or bottle-fed $(N=6)$ their infant during the study visits. We chose 8 -weeks postpartum for the TSST given that breastfeeding would be strongly established by this time point and to ensure that we captured women who had experienced symptoms of PPD. Venous blood samples were collected via antecubital IV.

\subsection{Psychometric measures}

\subsubsection{Edinburgh Postnatal Depression Scale (EPDS)—Edinburgh Postnatal} Depression Scale (EPDS) is a widely validated instrument developed and used to assess depression during pregnancy and postpartum (Cox et al., 1987). Participants completed the EPDS at enrollment, as well as 2- and 8-weeks postpartum. There have been multiple reports in the literature showing that the EPDS demonstrates both good specificity and sensitivity in identifying PPD (Gaynes et al., 2005). Scores of $\geq 12$ have been consistently shown to be correlated with a clinical diagnosis of major depressive disorder, when compared with a SCID (Cox et al., 1987). A cutoff score of $\geq 10$ was used as a positive screen in our study, as scores of 10-12 were associated with a diagnosis of minor depressive disorder (Cox et al., 1987; Gaynes et al., 2005).

2.1.2. The Spielberger State and Trait Anxiety Inventory (STAI)-The Spielberger State and Trait Anxiety Inventory (STAI), a validated instrument used to assess anxiety and validated for use in the perinatal population, (Meades and Ayers, 2011) was administered at enrollment during the third trimester, as well as at the 2- and 8-weeks postpartum visits. For the state inventory, a four-level Likert scale was used ranging from "not at all" to "very much so" to rate feelings in the moment, such as, "I feel nervous," "I feel calm," and "I feel content." For the trait inventory, a four-level Likert scale was also used ranging from 
"almost never" to "almost always" to rate statements such as "I have disturbing thoughts," "I feel satisfied with myself" and "I make decisions easily." State inventory captures perceived in-the-moment affect, while the trait inventory provides a stable measure of anxiety over time (Alpha (reliability) $=0.89$ to 0.92 and 0.90 to 0.94 , respectively) (Spielberger, 1983). Population normative data (women ages 19-39) for the Spielberger STAI shows the median scores for both state and trait anxiety to be 34 . We classified women as symptomatic with state scores greater than 34 . Breastfeeding intensity, defined as the proportion of feeds in the past week that were breast milk, and observed feeding sessions have been previously evaluated and described (Stuebe et al., 2013).

2.1.3. A modified version of The Trier Social Stress Test-A modified version of The Trier Social Stress Test was administered at the 2-month postpartum visit after a 10min period of baseline rest and a feeding session, followed by another 10-min rest prior to initiation of the TSST. The TSST has been shown to reliably induce large and consistent HPA and cardiovascular responses (Kirschbaum et al., 1993; Kudielka et al., 2007a,b). The test was modified, using the PASAT, which uses addition (described below), instead of the traditional PASST, which uses subtraction. The TSST consists of the following: Pre-Task Instructions: (5min) Subjects were introduced to 2 people (the 'selection committee') and asked to assume the role of a job applicant. Anticipation Period: The subject then was told to prepare a speech for $3 \mathrm{~min}$ in the presence of the committee. Speech: The committee then asked the subject to deliver her talk for $5 \mathrm{~min}$ while being video- and audio-recorded. If the subject finished early, the committee responded with prepared questions to ensure that each subject spoke for the entire $5 \mathrm{~min}$. These questions were designed to be non-harassing and to create a feeling of lack of predictability/controllability (e.g., "Do you have any enemies?") Paced Auditory Serial Addition Test (PASAT): The committee then used a recording of standardized instructions asking subjects to add single digits as quickly and accurately as possible for $5 \mathrm{~min}$. Subjects were given a new number every $3 \mathrm{~s}$ and asked to add this number to the number they had previously heard. Stress Recovery: The subject then sat quietly alone for $10 \mathrm{~min}$. During the TSST, study staff cared for the infant in a separate room.

\subsection{Neuroendocrine marker assessment}

At 8-weeks postpartum, venous blood samples were collected at baseline after 10min of rest prior to a feeding session (OT, CORT, CRH), as well as during minutes 1, 4, 7 and 10 of infant feeding (OT), followed by a sample taken $10 \mathrm{~min}$ post feed recovery (OT, CORT, CRH). Next, during administration of the TSST, blood samples were collected at minute 2 during the speech and minute 4 of the PASAT (OT, CORT and OT, CORT, CRH; Fig. 1). A final sample was collected 10min post stress recovery (OT, CORT, CRH), as HPA-axis responses are reliably found 10 to 30min following the TSST (Kirschbaum et al., 1993; Kudielka et al., 2007a,b). Each venous sample was collected into pre-chilled vacutaineri tubes, immediately cold-centrifuged, aliquoted into pre-chilled cryotubes and stored at $-80^{\circ}$ Centigrade (C) for later endocrine assays. The level of OT in EDTA-treated plasma, with aprotinin added to prevent peptide degradation, was determined by enzyme immunoassay (EIA) with extraction (Enzo Life Sciences, NY) as per previously described methods (Stuebe et al., 2013). The level of detection was $1.1 \mathrm{pg} / \mathrm{mL}$ with intra- and inter- 
assay cv of 4.6 and 8.7, respectively. We used commercial EIA to measure CORT. The level of detection was $0.7 \mu \mathrm{g} / \mathrm{dL}$ with intra- and inter-assay cv of 6.6 and 7.7, respectively. Neuroendocrine marker assessment at initial assessment and 2-wks postpartum has been described elsewhere (Stuebe et al., 2013).

\subsection{Statistical analysis}

Characteristics of the study population were compared using chi square tests or Fisher's Exact tests for categorical variables and analysis of variance or Kruskal-Wallis for continuous variables (Table 1).

Because OT levels vary due to pulsatile release, a composite variable was created for OT, using Area Under the Curve (AUC) (Pruessner et al., 2003) to reflect overall OT across the feeding session. We used a mixed models approach to repeated measures (MMRM) analysis using a Kenward-Roger approximation for the denominator degrees of freedom to quantify the association between subclinical anxiety and/or depression and each of three measures during the TSST: heart rate, OT, and CORT. Breastfeeding immediately prior to the TSST has been associated with a diminished CORT response (Heinrichs et al., 2001). Therefore, we further sought to test whether OT during breastfeeding was associated with HPA axis activity during the TSST. For this analysis, we used repeated measures analysis to model the effect of OT AUC during feeding, time, symptom group, and their interactions, on CORT levels during the TSST. We hypothesized that a smaller OT AUC would be associated with an exaggerated HPA axis stress response to the TSST. To explore whether maternal history of depression or anxiety affected the relationship between OT AUC and HPA response, we similarly modeled interactions between OT AUC, time, and SCID-verified history of depression or anxiety. Residuals were tested for normality, and, if non-normal, neuroendocrine markers were log-transformed.

\section{Results and discussion}

We recruited 52 women during the third trimester of pregnancy with intention to breastfeed for at least 3 months. Of those women, 47 completed all 3 visits (Fig. 2). Of these 23 had no history of depression or anxiety, 16 had a lifetime history of depression or anxiety and 8 were currently depressed or anxious and met criteria for postpartum depression based on diagnostic interview at initial time of enrollment. These 3 groups have been previously described (Stuebe et al., 2013) and were similar in age, race/ethnicity, income and education (Table 1).

Of the 47 women who completed the TSST, 39 were classified as "breastfeeding" during the study visit; 38 fed at breast and 1 expressed milk. Of these 39 women, 11 had mild depression/anxiety symptoms (EPDS $\geq 10$ and/or STAI state $\geq 34$ ). Eight women were classified as not breastfeeding; 6 formula-fed and 2 held their infants during this visit. Of these 8 women, 3 had mild depression/anxiety symptoms. Six of these 8 women reported breastfeeding or pumping at least once in the week prior to the study visit. Among women who did not breastfeed during the study visit, breastfeeding intensity over the past week was markedly lower than among women who breastfed during the study visit (12 vs. $100 \%$, $p<0.01$, Table 1). Two of these women had not breastfed or pumped in the week prior to the 
study visit. Because our sample size was small, we analyzed all mothers who did not breastfeed during the visit together. Our overarching goal was to quantify stress reactivity following a breastfeeding episode among symptomatic versus asymptomatic women, while women who had not breastfed served as a comparison group.

Compared with mothers who breastfed during the visit, women who did not breastfeed had lower levels of OT during a feeding session (group $F(1,45)=6.74, p=0.01$ and group $\times$ time $F(5,40.5)=6.3, p<0.01)$ and greater increases in CORT $($ group $F(1,41.7)=5.71, p=$ 0.02 and group $\times$ time $F(3,40)=7.91, p<0.01)$ and heart rate $(\mathrm{HR})$ (group $\times$ time $F(15,208)$ $=2.64, p<0.01$ ) during administration of the TSST (Fig. 3). As we have previously reported, breastfeeding mothers with depression/anxiety symptoms (EPDS $>10$ and/or STAI state $\geq$ 34) had lower levels of OT during feeding than breastfeeding mothers without symptoms (group $F(1,37.6)=4.26, p<0.05$ ). During the TSST, breastfeeding mothers with symptoms had greater HR (group $\times$ time $F(15,158)=1.91, p=0.03$ ) and non-significantly higher CORT levels throughout the TSST (group $F(1,33.5)=3.31, p=0.08$ ), as compared with asymptomatic breastfeeding mothers. When we compared breastfeeding mothers with and without SCID-verified histories of depression and anxiety, we did not find significant differences in OT and CORT levels.

Previous studies have demonstrated that OT blunts HPA axis activation, and breastfeeding buffers CORT response to stress (Heinrichs et al., 2001; Heinrichs et al., 2003). To determine whether the relationship between OT and CORT varied among breastfeeding women with and without symptoms in our sample, we tested for interactions among OT AUC during feeding, mood symptom group, and time to predict CORT during the TSST (Fig. 4). A significant group $\times$ OT AUC $\times$ time interaction was observed $(F(7.39 .8)=2.26$, $p<0.05)$. Among asymptomatic breastfeeding women, higher OT AUC was associated with marginally lower CORT levels during the TSST. In contrast, among symptomatic breastfeeding women, higher OT AUC was paradoxically associated with higher CORT levels during the TSST. We then tested whether a SCID-verified past or current depression or anxiety episode $(N=18)$ vs. a negative SCID $(N=21)$ modified the association between OT AUC and CORT during the TSST. We did not find evidence of an interaction. These results suggest that among women with current symptoms, OT increases, rather than decreases, CORT response to stress.

\section{Conclusions}

In a longitudinal, perinatal prospective cohort study, we found that both infant feeding method and maternal mood symptoms were associated with differences in response to a standardized social stressor. Compared with women who had bottle-fed their infants, women who had breastfed had lower CORT secretion and lower HR during a standardized social stressor. Of those women who breastfed, those with symptoms of anxiety and depression had significantly higher levels of CORT and higher HR during stress when compared to the asymptomatic women.

Our results confirm and extend earlier work demonstrating HPA axis dysregulation in both major depressive disorder (MDD) outside of the perinatal period as well as PPD (Jolley et 
al., 2007). Furthermore, our results confirm earlier work indicating dysregulated patterns of OT release in men and women with MDD, with higher levels of OT associated with higher levels of CORT (Cyranowski et al., 2008; Parker et al., 2010). Chen et al. (2011) have found that men with different OT receptor genotypes (OXTR) have a differential stress response following social support, with a particular polymorphism serving a protective effect, seen as a decrease in CORT levels. Clinical studies similarly suggest that OT may not improve mood among women with affective symptoms. In a cohort of women with symptoms of PPD, Mah et al. (2013) found that administration of intranasal OT made mothers sadder; however, these mothers described their relationship with their infant more positively. In contrast, Zelkowitz et al. (2014) found that OT was protective in women with high psychosocial stress levels; higher endogenous OT levels were associated with fewer depressive symptoms and more sensitive maternal behavior in a small cohort.

These studies suggest that OT plays a critical role in maternal behavior. Further, it is possible that OT plays a role in buffering the stress response in breastfeeding women; however, this relationship appears to be disturbed in women with PPD, suggesting that dysregulation of OT secretion and/or response to OT may play a role in the pathogenesis of PPD (Heinrichs et al., 2001, 2003). Our results demonstrate that although breastfeeding was associated with buffering of stress-induced CORT, symptomatic breastfeeding women experienced a positive correlation between OT AUC and CORT, instead of the expected negative correlation, seen among asymptomatic women.

Our findings must be interpreted within the context of our study design. We did not measure $\mathrm{ACTH}$ and therefore cannot determine whether OT affects CORT secretion via ACTH or via direct effects on the adrenal. In addition, because a small number of women did not breastfeed during the study visit, we were not able to analyze differences in TSST response among non-breastfeeding women by symptom group. However, the proportion of symptomatic women in the breastfeeding and non-breastfeeding groups were similar (Bottle: $3 / 8,38 \%$ vs. breast $11 / 39,28 \%$, Fisher's Exact $p=0.68$ ). Our non-breastfeeding group included women who were still lactating, although the intensity of lactation among these women was low. Prior work among euthymic, exclusively breastfeeding women found that breastfeeding immediately before the TSST has a greater impact on the HPA axis than infant contact alone (Heinrichs et al., 2001). Further studies are needed to explore how both overall lactation intensity and affective symptoms moderate acute associations between infant contact, breastfeeding, and HPA axis response. Moreover, our sample size is relatively small, and participants had mild symptoms of depression and anxiety, limiting our power to detect differences in neuroendocrine markers. Specifically, due to the lower EPDS scores in our population, we were unable to adequately examine the effect of EPDS $\geq 10$ on measures, because only two of the breastfeeding participants scored in this range. Nevertheless, we detected differences in the relationship between OT and CORT among women with higher vs. lower state anxiety. Furthermore, we did not detect any significant association between history of previous episodes of depression or anxiety and greater risk for HPA axis dysregulation, thereby decreasing the likelihood that prior histories of depression confound our results and supporting our hypothesis that current anxiety and depressive symptoms are the driving factor. Given the role of stress reactivity in the pathogenesis of depression, our 
data suggest that dysregulation of OT modulation of CORT response to stressors may play a role in the pathogenesis of PPD. However, we cannot determine the direction of this association in an observational study; it is also possible that PPD may lead to dysregulation of OT. We believe our study is the first to examine this relationship between lactation, OT and the HPA axis in postpartum women with depression and anxiety symptoms.

In conclusion, we found that mild symptoms of depression and anxiety modify the relationship between OT and CORT response to stress during the peripartum period. While breastfeeding buffered the stress response, irrespective of mood and anxiety symptoms, the association between OT and CORT was altered in symptomatic women. Increased OT was associated with an increase - instead of the expected decrease - in CORT levels in women with mild depression and anxiety. Our findings suggest that dysregulation of OT and the HPA axis may contribute to postpartum symptoms of depression and anxiety among breastfeeding women.

\section{Acknowledgments}

Role of the funding source

1. UL1RR025747(50KR20957) - to S. Meltzer-Brody and A. Stuebe

2. K23 MH085165-01A1 -- to S. Meltzer-Brody

3. R01HD073220 -- to A. Stuebe

4. P01DA022446 -- K. Grewen

5. T32 MH93315 - to D. Rubinow

We would like to thank all of the participants who volunteered their time to our study.

\section{References}

AHRQ. Breastfeeding and Maternal and Infant Health Outcomes in Developed Countries. Department of Health and Human Services; 2007.

American Psychiatric Association. Diagnostic and Statistical Manual of Mental Disorders. American Psychiatric Publishing; Arlington, VA: 2013.

Aragona B, Liu Y, Yu Y, Curtis J, Detwiler J, Insel T, Wang Z. Nucleus accumbens dopamine differentially mediates the formation and maintenance of monogamous pair bonds. Nat Neurosci. 2006; 9:133-139. [PubMed: 16327783]

Bernstein IH, et al. Symptom features of postpartum depression: are they distinct? Depress Anxiety. 2008; 25:20-26. [PubMed: 17187349]

Bloch M, Daly R, Rubinow D. Endocrine factors in the etiology of postpartum depression. Comp Psychiatry. 2003; 44:234-246.

Bloch M, Rubinow D, Schmidt P, Lotsikas A, Chrousos G, Cizza G. Cortisol response to ovine corticotropin-releasing hormone in a model of pregnancy and parturition in euthymic women with and without a history of postpartum depression. J Clin Endocrinol Metab. 2005; 90:695-699. [PubMed: 15546899]

Chen FS, Kumsta R, et al. Common oxytocin receptor gene (OXTR) polymorphism and social support interact to reduce stress in humans. Proc Natl Acad Sci USA. 2011; 108(50):19937-19942. [PubMed: 22123970]

Cox J, Holden J, Sagovsky R. Detection of postnatal depression. Development of the 10-item Edinburgh Postnatal Depression Scale. Br J Psychiatry. 1987; 150:782-786. [PubMed: 3651732] 
Cyranowski JM, Hofkens TL, Frank E, Seltman H, Cai HM, Amico JA. Evidence of dysregulated peripheral oxytocin release among depressed women. Psychosom Med. 2008; 70(9):967-975. [PubMed: 19005082]

Dennis C, McQueen K. The relationship between infant-feeding outcomes and postpartum depression: a qualitative systematic review. Pediatrics. 2009; 123(4):736-751.

Figueiredo B, Dias C, Brandao S, Canario C, Nunes-Costa R. Breastfeeding and postpartum depression: state of the art review. J Pediatr (Rio J). 2013; 89(4):332-338. [PubMed: 23791236]

First, MB.; Spitzer, RL.; Gibbon, M.; Williams, JB. Structured Clinical Interview for DSM-IV-TR Axis I Disorders, Research Version, Non-Patient Edition (SCID-I/NP). N. Y S. P. I. Biometrics Research; New York, NY: 2002.

Flynn H, Davis M, Marcus S, Cunningham R, Blow F. Rates of maternal depression in pediatric emergency department and relationship to child service utilization. Gen Hosp Psychiatry. 2004; 26(4):316-322. [PubMed: 15234828]

Gaynes B, Gavin N, Meltzer-Brody S. Perinatal depression: prevalence, screening accuracy and screening outcomes. Evid Rep Technol Assess. 2005; 119:1-8.

Greenwood J, Parker G. The dexamethasone suppression test in the puerperium. Aust NZ J Psychiatry. 1984; 18:282-284.

Groer M, Davis M. Cytokines, infections, stress and dysphoric moods in breastfeeders and formula feeders. J Obstet Gynecol Neonatal Nurs. 2006; 35(5):599-607.

Heinrichs M, et al. Social support and oxytocin interact to suppress cortisol and subjective responses to psychosocial stress. Biol Psychiatry. 2003; 54(12):1389-1398. [PubMed: 14675803]

Heinrichs M, Meinlschmidt G, Neumann I, Wagner S, Kirschbaum C, Ehlert U, Hellhammer D. Effects of suckling on hypothalamic_-pituitary—adrenal axis responses to psychosocial stress in postpartum lactating women. J Clin Endocrinol Metab. 2001; 86(10):4798-4804. [PubMed: 11600543]

Jolley S, Elmore S, Barnard K, Carr D. Dysregulation of the hypothalamic-pituitary-adrenal axis in postpartum depression. Biol Res Nurs. 2007; 8:210-222. [PubMed: 17172320]

Jonas W, Mileva-Seitz V, Girard A, Bisceglia R, Kennedy J, Sokolowski M, Meaney M, Fleming A, Steiner M, Team MR. Genetic variation in oxytocin rs2740210 and early adversity associated with postpartum depression and breastfeeding duration. Genes Brain Behav. 2013; 12(7):681-694. [PubMed: 23941164]

Kendall-Tackett K, Cong Z, Hale T. Depression, sleep quality and maternal well-being in postpartum women with a history of sexual-assault: a comparison of breastfeeding, mixed-feeding and formula-feeding mothers. Breastfeed Med. 2013; 8(1):16-22. [PubMed: 23249130]

Kirschbaum C, Pirke K, Hellhammer D. The 'Trier Social Stress Test'-a tool for investigating psychobiological stress responses in a laboratory setting. Neuropsychobiology. 1993; 28(1-2):7681. [PubMed: 8255414]

Kudielka B, Hellhammer D, Kirschbaum C. Ten years of research with the Trier Social Stress Test (TSST). Social Neuroscience; Integrating Biological and Psychological Explanations of Social Behavior. 2007:56-83.

Kudielka, B.; Hellhammer, D.; Kirschbaum, C. Ten years of research with the Trier Social Stress Test (TSST)—revisited. In: Harmon-Jones, E.; Winkilman, P., editors. Social Neuroscience. Guilford Press; New York, NY: 2007b.

Lindahl V, Pearson J, Colpe L. Prevalence of suicidality during pregnancy and postpartum. Arch Womens Ment Health. 2005; 8(2):77-87. [PubMed: 15883651]

Lonstein J, Stern J. Site and behavioral specificity of periaqueductal gray lesions on postpartum sexual, maternal, and aggressive behaviors in rats. Brain Res. 1998; 804:21-35. [PubMed: 9729249]

Magiakou M, Mastorakos G, Rabin D, Dubbert B, Gold P, Chrousos G. Hypothalamic corticotropinreleasing hormone suppression during the postpartum period: implications for the increase in psychiatric manifestations at this time. J Clin Endocrinol Metab. 1996; 81:1912-1917. [PubMed: 8626857] 
Mah BL, Ijzendoorn MHV, Smith R, Bakermans-Kranenburg MJ. Oxytocin in postnatally depressed mothers: its influence on mood and expressed emotion. Prog Neuropsychopharmacol Biol Psychiatry. 2013; 40:267-272. [PubMed: 23085508]

Meades R, Ayers S. Anxiety measures validated in perinatal populations: a systematic review. J Affect Disord. 2011; 133(1-2):1-15. [PubMed: 21078523]

Meinlschmidt G, Heim C. Sensitivity to intranasal oxytocin in adult men with early parental separation. Biol Psychiatry. 2007; 61(9):1109-1111. [PubMed: 17141739]

Numan M, Numan M, Pliakou N, Stolzenberg D, Mullins O, Murphy J, Smith C. The effects of D1 or D2 dopamine receptor antagonism in the medial preoptic area, ventral pallidum, or nucleus accumbens on the maternal retrieval response and other aspects of maternal behavior in rats. Behav Neurosci. 2005; 119:1588-1604. [PubMed: 16420162]

Pang W, Hartmann P. Initiation of human lactation: secretory differentiation and secretory activation. J Mammary Gland Biol Neoplasia. 2007; 12:211-221. [PubMed: 18027076]

Parker KJ, Kenna HA, Zeitzer JM, Keller J, Blasey CM, Amico JA, Schatzberg AF. Preliminary evidence that plasma oxytocin levels are elevated in major depression. Psychiatry Res. 2010; 178(2):359-362. [PubMed: 20494448]

Paul I, Downs D, Schaefer E, Beiler J, Weisman C. Postpartum anxiety and maternal-infant health outcomes. Pediatrics. 2013; 131:e1218-e1224. [PubMed: 23460682]

Pedersen C. Oxytocin control of maternal behavior. Regulation by sex steroids and offspring stimuli. Ann Acad Sci. 1997; 807:126-145.

Pedersen C, Caldwell J, Walker C, Ayers G, Mason G. Oxytocin activates the postpartum onset of maternal behavior in the ventral tegmental and medial preoptic areas. Behav Neurosci. 1994; 108:1163-1171. [PubMed: 7893408]

Pruessner JC, Kirschbaum C, Meinlschmid G, Hellhammer DH. Two formulas for computation of the area under the curve represent measures of total hormone concentration versus time-dependent change. Psychoneuroendocrinology. 2003; 28(7):916-931. [PubMed: 12892658]

Putnam K, Robertson-Blackmore E, Sharkey K, Payne J, Bergink V, Munk-Olsen T, Deligiannidis K, Altemus M, Newport D, Apter G, Devouche E, Vikorin A, Magnusson P, Lichenstein P, Penninx B, Buist A, Bilszta J, O’Hara M, Stuart S, Brock R, Roza S, Tiemeier H, Guille C, Epperson C, Kim D, Schmidt P, Martinez P, Wisner K, Stowe Z, Jones I, Rubinow D, Sullivan P, MeltzerBrody S. Heterogeneity of Postpartum Depression: A latent class analysis. The PACT consortium: postpartum depression-action towards causes and treatment. Lancet Psychiatry. In press

Shahrokh DK, Zhang TY, Diorio J, Gratton A, Meaney MJ. Oxytocin—dopamine interactions mediate variations in maternal behavior in the rat. Endocrinology. 2010; 151(5):2276-2286. [PubMed: 20228171]

Spielberger, C. Manual for the State-Trait Anxiety Inventory. Consulting Psychologists Press; Palo Alto, CA: 1983.

Stuebe A, Grewen K, Meltzer-Brody S. Association between maternal mood and oxytocin response to breastfeeding. J Womens Health. 2013; 22(4):352-361.

Stuebe A, Horton B, Chetwynd E, Watkins S, Grewen K, Meltzer-Brody S. Prevalence and risk factors for early, undesired weaning attributed to lactation dysfunction. J Womens Health. 2014; 23(5): 404-412.

Thombs BD, Arthurs E, Coronado-Montoya S, Roseman M, Delisle VC, Leavens A, Levis B, Azoulay L, Smith C, Ciofani L, Coyne JC, Feeley N, Gilbody S, Schinazi J, Stewart DE, Zelkowitz P. Depression screening and patient outcomes in pregnancy or postpartum: a systematic review. $\mathrm{J}$ Psychosom Res. 2014; 76:433-446. [PubMed: 24840137]

Wisner K, Stowe Z. Psychobiology of postpartum mood disorders. Semin Reprod Endocrinol. 1997; 15:77-89. [PubMed: 9065980]

Zelkowitz P, Gold I, Feeley N, Hayton B, Carter C, Tulandi T, Abenhaim H, Levin P. Psychosocial stress moderates the relationships between oxytocin, perinatal depression, and maternal behavior. Horm Behav. 2014 


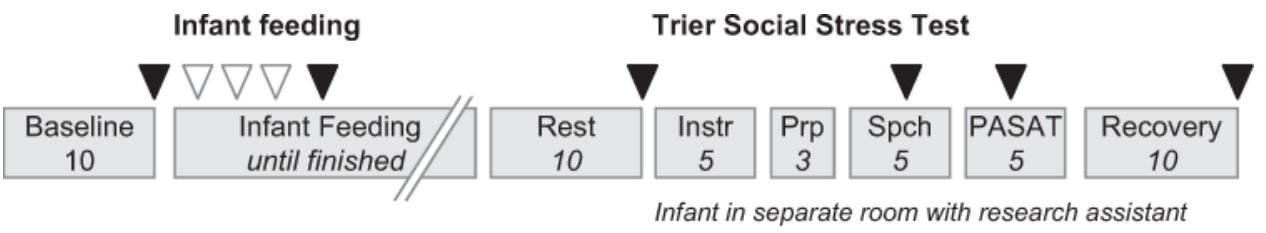

Maternal blood sample, via antecubitalIV

$\nabla$ Oxytocin, Cortisol $\nabla$ Oxytocin

Task
Time, minutes

Fig. 1.

Eight week postpartum visit protocol. 


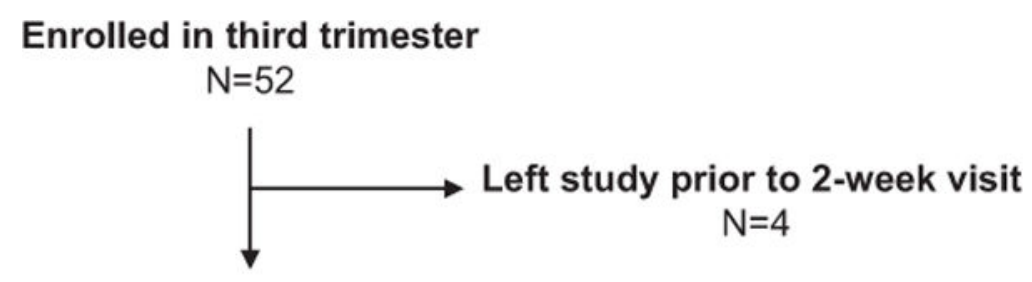

Completed 2-week visit

$\mathrm{N}=48$

\section{Completed 8-week visit}

$\mathrm{N}=47$

Fig. 2.

Study participant flow chart. 

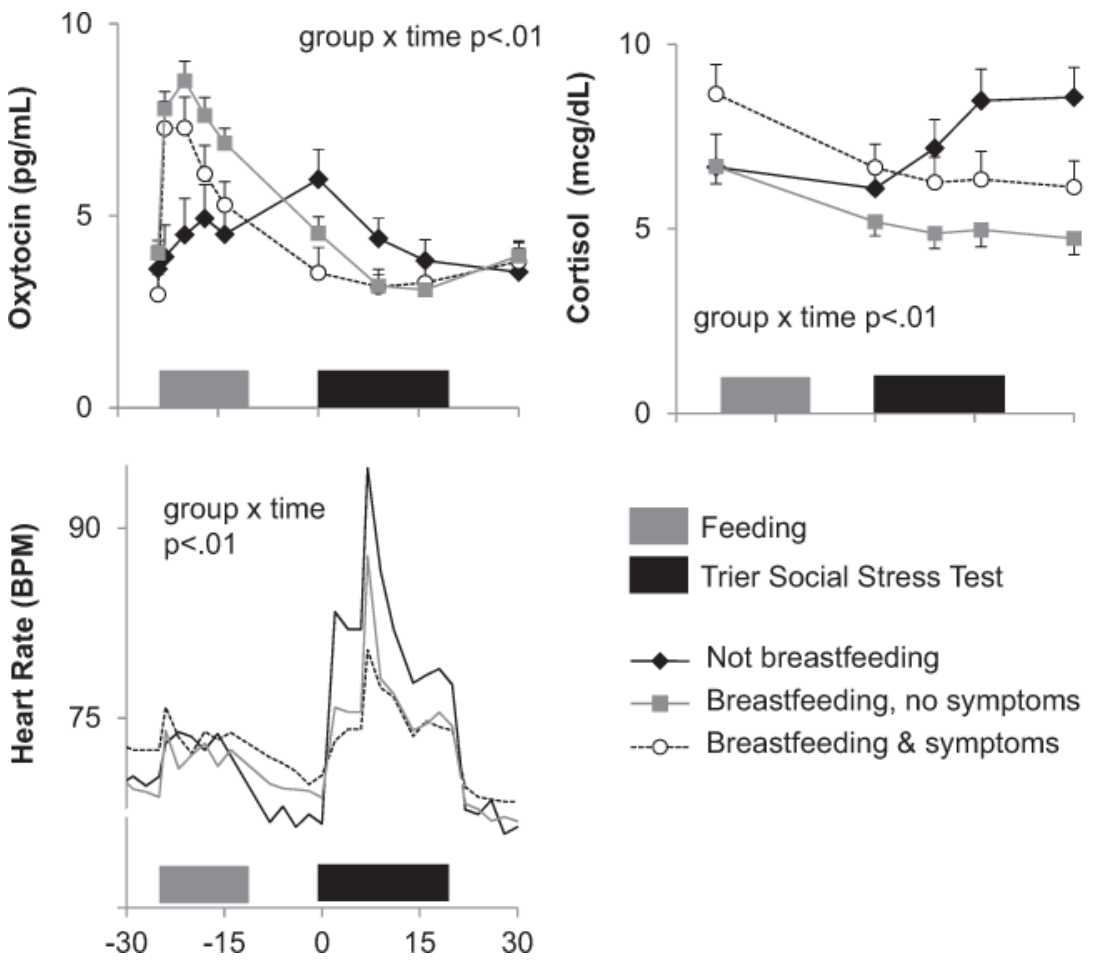

--o-- Breastfeeding \& symptoms

Fig. 3.

Neuroendocrine profile during study visit by affective symptoms and feeding. 

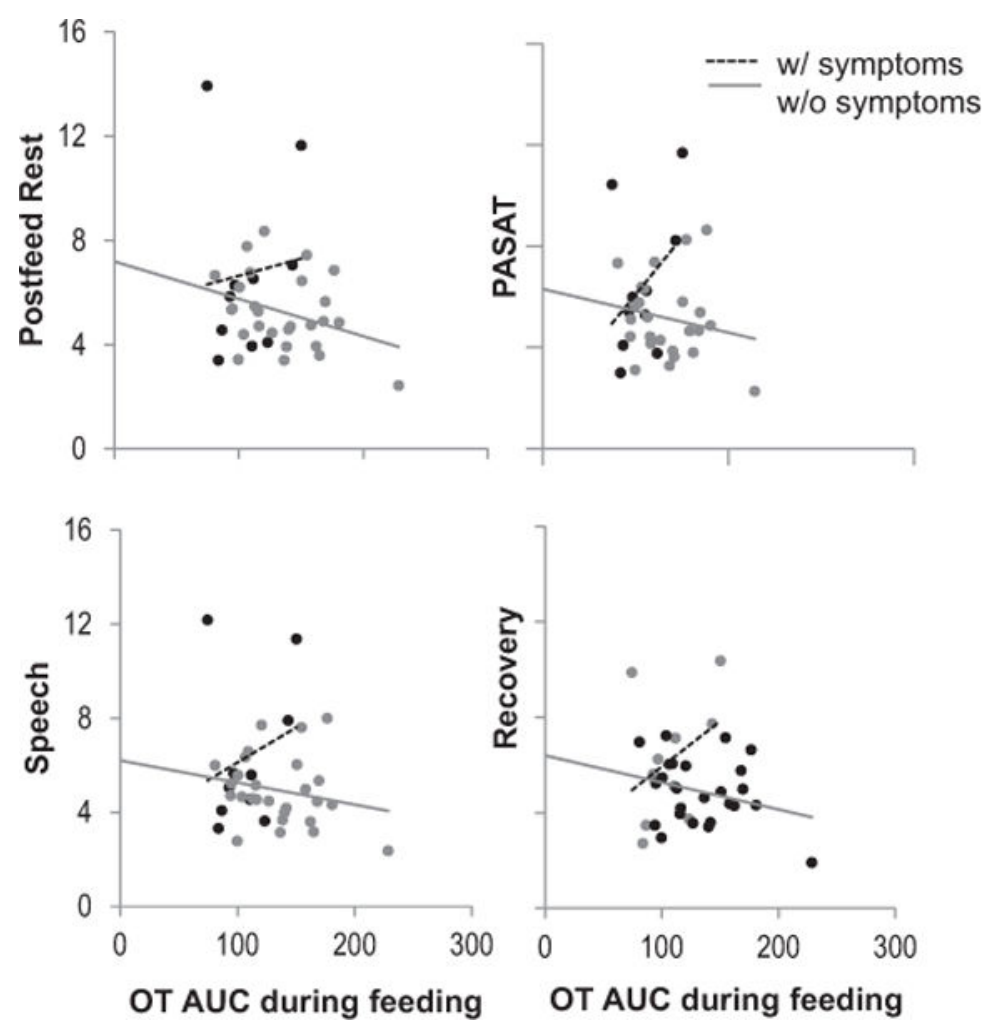

Fig. 4.

Association between oxytocin during feeding and cortisol during TSST, among women breastfeeding at study visit. OT AUC during breastfeeding and cortisol during TSST among women with and without mood symptoms at 8 weeks postpartum. (Repeated measures group $\times$ time $\times$ OT AUC $p<0.05)$. 


\section{Table 1}

Characteristics of study population by feeding method during study visit and mood symptoms at 8 weeks postpartum.

\begin{tabular}{|c|c|c|c|c|}
\hline & $\begin{array}{l}\text { Breastfeeding, no symptoms } \\
\text { Mean (SD) }\end{array}$ & $\begin{array}{l}\text { Breastfeeding, with } \\
\text { symptoms } \\
\text { Mean (SD) }\end{array}$ & $\begin{array}{l}\text { Not breastfeeding } \\
\text { Mean (SD) }\end{array}$ & $p^{*}$ \\
\hline$N$ & 28 & 11 & 8 & \\
\hline \multirow[t]{2}{*}{ Age (years) } & $32.1(5.0)$ & $32.0(2.8)$ & $29.5(6.0)$ & 0.40 \\
\hline & $N(\%)$ & $N(\%)$ & $N(\%)$ & \\
\hline Race & & & & 0.16 \\
\hline White & $23(82.1)$ & $11(100)$ & $5(62.5)$ & \\
\hline Black or African-American & $4(14.3)$ & $0(0.0)$ & $3(37.5)$ & \\
\hline Asian & $1(3.6)$ & $0(0.0)$ & $0(0.0)$ & \\
\hline Education & & & & 0.15 \\
\hline$<4 \mathrm{yr}$ college graduate & $3(10.7)$ & $1(9.1)$ & $4(50.0)$ & \\
\hline Graduated 4 yr college & $9(32.1)$ & $3(27.3)$ & $2(25.0)$ & \\
\hline Post graduate & $16(57.1)$ & $7(63.6)$ & $2(25.0)$ & 0.80 \\
\hline \multicolumn{5}{|l|}{ BMI } \\
\hline $\mathrm{BMI}<25$ & $11(39.3)$ & $2(18.2)$ & $2(25.0)$ & \\
\hline BMI $25<30$ & $11(39.3)$ & $6(54.5)$ & $4(50.0)$ & \\
\hline BMI $30+$ & $6(21.4)$ & $3(27.3)$ & $2(25.0)$ & \\
\hline Income & & & & 0.68 \\
\hline Less than $\$ 40,000$ & $6(22.2)$ & $1(9.1)$ & $3(37.5)$ & \\
\hline$\$ 40,000-59,999$ & $5(18.5)$ & $1(9.1)$ & $1(12.5)$ & \\
\hline$\$ 60,000-99,999$ & $7(25.9)$ & $5(45.5)$ & $3(37.5)$ & \\
\hline$\$ 100,000$ or above & $9(33.3)$ & $4(36.4)$ & $1(12.5)$ & \\
\hline Nulliparous & $15(53.6)$ & $5(45.5)$ & $6(75.0)$ & 0.47 \\
\hline Mode of delivery & & & & 0.25 \\
\hline Spontaneous vaginal birth & $21(87.5)$ & $8(72.7)$ & $4(66.7)$ & \\
\hline Vacuum & $1(4.2)$ & $2(18.2)$ & $0(0.0)$ & \\
\hline C-section & $2(8.3)$ & $1(9.1)$ & $2(33.3)$ & \\
\hline \multicolumn{5}{|l|}{ Mood symptoms } \\
\hline Prenatal anxiety & $7(25.0)$ & $5(45.5)$ & $2(25.0)$ & 0.45 \\
\hline Anxiety at 2 weeks & $4(14.3)$ & $9(81.8)$ & $2(25.0)$ & $<0.01$ \\
\hline Anxiety at 8 weeks & $0(0.0)$ & $11(100)$ & $2(25.0)$ & $<0.01$ \\
\hline Prenatal depression & $1(3.6)$ & $1(9.1)$ & $1(12.5)$ & 0.36 \\
\hline Depression at 2 weeks (EPDS $>10$ ) & $2(7.1)$ & $5(45.5)$ & $1(12.5)$ & 0.01 \\
\hline Depression at 8 weeks (EPDS $>10$ ) & $0(0.0)$ & $2(18.2)$ & $2(25.0)$ & 0.02 \\
\hline Depression/anxiety history by SCID & & & & $<0.01$ \\
\hline Never & $20(71.4)$ & $1(9.1)$ & $2(25.0)$ & \\
\hline Lifetime, not current & $5(17.9)$ & $6(54.5)$ & $5(62.5)$ & \\
\hline Current & $3(10.7)$ & $4(36.4)$ & $1(12.5)$ & \\
\hline
\end{tabular}




\begin{tabular}{llll}
\hline & $\begin{array}{l}\text { Breastfeeding, no symptoms } \\
\text { Mean (SD) }\end{array}$ & $\begin{array}{l}\text { Breastfeeding, with } \\
\text { symptoms } \\
\text { Mean (SD) }\end{array}$ & $\begin{array}{l}\text { Not breastfeeding } \\
\text { Mean (SD) }\end{array}$ \\
\hline $\begin{array}{l}\text { Breastfeeding intensity, 7 days prior to } \\
\text { study visit, median (IQR) }\end{array}$ & $100 \%$ (IQR 100-100\%) & $100 \%$ (IQR 100-100\%) & $12 \%$ (IQR 1-29\%) \\
\hline
\end{tabular}

* ANOVA for continuous variables, Fisher's Exact Test for categorical variables, Kruskal-Wallis for breastfeeding intensity. 